\title{
Heartbeat instability as auto-oscillation between dim and bright void regimes
}

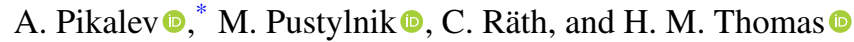 \\ Institut für Materialphysik im Weltraum, Deutsches Zentrum für Luft- und Raumfahrt e. V. (DLR), 82234 Weßling, Germany
}

(Received 9 April 2021; revised 30 August 2021; accepted 4 October 2021; published 26 October 2021)

\begin{abstract}
We investigated the self-excited as well as optogalvanically stimulated heartbeat instability in RF discharge complex plasma. Three video cameras measured the motion of the microparticles, the plasma emission, and the laser-induced fluorescence simultaneously. Comprehensive studies of the optogalvanic control of the heartbeat instability revealed that the microparticle suspension can be stabilized by a continuous laser, whereas a modulated laser beam induces the void contraction either transiently or resonantly. The resonance occurred when the laser modulation frequency coincided with the frequency of small breathing oscillations of the microparticle suspension, which are known to be a prerequisite to the heartbeat instability. Based on the experimental results we suggest that the void contraction during the instability is caused by an abrupt void transition from the dim to the bright regime [Pikalev et al., Plasma Sources Sci. Technol. 30, 035014 (2021)]. In the bright regime, a time-averaged electric field at the void boundary heats the electrons causing bright plasma emission inside the void. The dim void has much lower electric field at the boundary and exhibits therefore no emission feature associated with it.
\end{abstract}

DOI: 10.1103/PhysRevE.104.045212

\section{INTRODUCTION}

Complex or dusty plasma is a medium containing ionized gas and micron-sized solid particles [1-3]. The strongly coupled subsystem of such microparticles can be used for particle resolved studies of generic classical phenomena in condensed matter. For such studies, homogeneous microparticle suspensions are desirable. However, even microgravity does not guarantee homogeneous distribution and stability of the microparticle component. A void, i. e., a microparticlefree area, is one of most common disturbances of the complex plasma homogeneity under microgravity conditions. The void formation and growth also determine the nanoparticle generation cycle in plasma reactors [4].

Due to its small charge-to-mass ratio and its mesoscopic nature, the dust component introduces a variety of instabilities including dust density waves [5], transverse instability [6], filamentary mode and the void rotation [7-9], plasmoids and carousel instability $[10,11]$. Because of their multi-time-scale character, the instabilities in complex plasma pose a hard problem for numerical studies. The physical mechanism of most of these instabilities is still unknown.

In the first microgravity experiments with complex plasmas, a spontaneous periodic contraction of the void boundary was reported [12]. Because of its characteristic appearance as well as due to very low repetition frequency (from single contractions to hundreds $\mathrm{Hz}$ ), this phenomenon was termed "heartbeat" instability. This particular instability is the subject of the present work.

The heartbeat instability is a complex auto-oscillation phenomenon, which was treated in Ref. [13] as mixed-mode oscillations. Mixed-mode oscillations occur when a dynamical system switches between fast and slow motion and small

\footnotetext{
*Aleksandr.Pikalev@dlr.de
}

and large amplitude [14]. The mixed-mode oscillations are encountered in a wide range of phenomena including glow discharges [15,16], chemical processes [17,18], models of neuronal activity [19] and the real mammal heartbeat [20].

The heartbeat instability was investigated in microgravity conditions on board the International Space Station [21,22], in experiments with grown nanoparticles [13,23-25] and with the thermophoretic gravity compensation [26]. These experiments showed that oscillations of the discharge electrical parameters and the plasma emission are inherent in the heartbeat instability. Just before every void contraction, a short bright flash appears in the void. In some experiments, small oscillations of the plasma emission and the electrical signals occurs between the void contractions [13,24]. The heartbeat instability is sensitive to the amount and size of the microparticles, the gas pressure, and the discharge power making it difficult to reproduce the instability quantitatively. Such phenomena as "trampoline effect" [27] and "delivery instability" [28] seem to be qualitatively similar to the heartbeat instability. In Ref. [26], the heartbeat instability was resonantly stimulated by modulating the ionization rate with a tunable laser. It remained, however, unclear, which oscillatory mode of the void was excited by the tunable laser.

The heartbeat instability represents a very complex process involving the microparticle-plasma interactions. Despite several attempts to explain the heartbeat instability theoretically $[21,26,29]$, its physical mechanism is still unclear. Understanding of this phenomenon is essential for the physics of microparticle-plasma interactions and the void formation. It needs a comprehensive model of the microparticle dynamics coupled with plasma conditions. For such a model, understanding of the void formation mechanism is required.

It is commonly supposed that the void in complex plasmas is formed due to a balance between the electrostatic and the ion drag forces with the ion flow from the void into the 

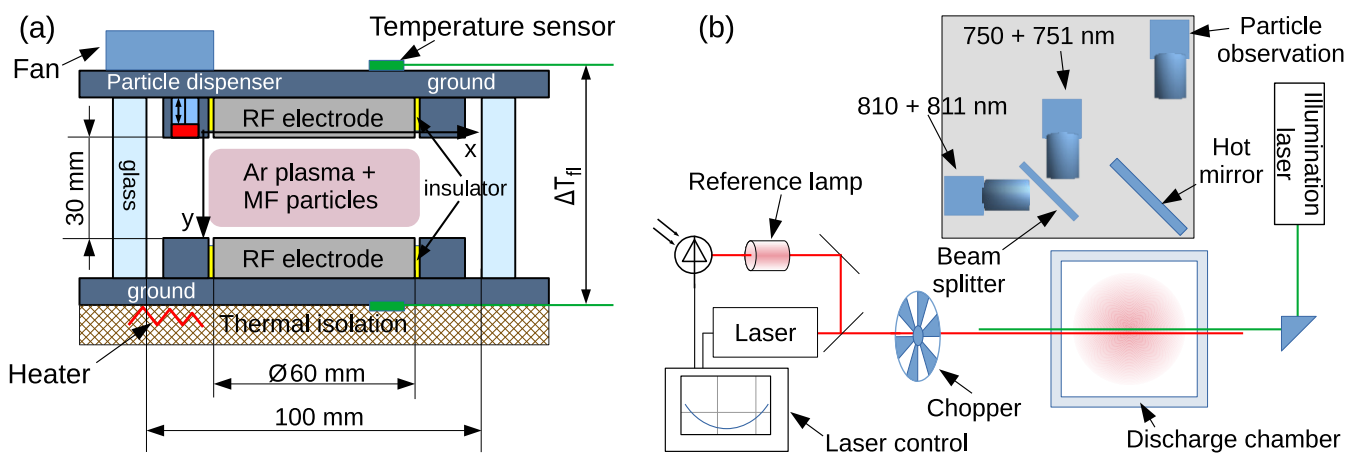

FIG. 1. Scheme of the experimental setup, (a) side view, (b) top view. The heart of the experiment is the PK-3 Plus chamber [34]. The bottom flange of the chamber can be heated to control the temperature gradient between the electrodes. The cameras observe the microparticle motion and plasma emission. The reference Ar lamp is used to control the wavelength of the tunable laser.

microparticle suspension [27,29-31]. However, this mechanism cannot explain the void formation at low discharge powers [32,33]. The experiment and the model in Ref. [33] demonstrated that the void can exist in one of two regimes depending on the discharge power. The so-called bright void regime corresponds to the conditions with higher RF discharge power and is characterized by the bright emission inside the void. In this case, strong time-averaged electric field at the top and the bottom void boundaries heats up the electrons enhancing the ionization and the emission. The mechanical stability of the boundary is in this case indeed determined by the balance of electrostatic and ion drag forces. The dim void regime corresponds to relatively low discharge power conditions and is characterized by the diffuse emission profile inside and around the void. The transition between the regimes has a discontinuous character. After the void formation, its size is almost independent of the discharge power in the dim regime, but the bright void grows rapidly with the discharge power increase after the transition. A simplified time-averaged one-dimensional (1D) fluid model in Ref. [33] could reproduce the dim void only when the radial ion losses were artificially introduced into the microparticle-free region of the plasma. In this case, the force balance on the top and bottom void boundaries could be satisfied for low plasma density and with much weaker time-averaged electric field.

Combining the comprehensive investigation of the optogalvanic control of the heartbeat instability with the concept of dim and bright void, we decompose the entire heartbeat process into several stages, which can be investigated separately.

\section{EXPERIMENT}

The experiments were conducted in the ground-based PK-3 Plus chamber (see Fig. 1) [34]. The plasma was produced by means of a capacitively coupled RF discharge. Two electrodes were driven in a push-pull mode by a sinusoidal signal with the frequency of $13.56 \mathrm{MHz}$. We used two types of melamine formaldehyde spheres with the diameters of $1.95 \mu \mathrm{m}$ and $2.15 \mu \mathrm{m}$ as microparticles. During a particular experiment, the microparticles of one of these types were injected into the discharge by an electromagnet-driven dispenser through a sieve.

Argon was fed into the chamber with 3 -sccm gas flow during the entire experiment. We mainly investigated the heartbeat instability with the gas pressure of $36 \mathrm{~Pa}$. We also observed the heartbeat instability at the pressures of 20 and $55 \mathrm{~Pa}$. In the case of lower pressure, other instabilities [7,8,11] made the discharge behavior complex and difficult to interpret. In the case of higher pressure, gas friction made the heartbeat less pronounced.

Under ground laboratory conditions, the microparticles concentrate themselves in the vicinity of the lower electrode. To obtain large volumetric microparticle suspensions, we compensated the gravitational force by means of thermophoresis [35]. The temperature gradient between the electrodes was controlled by heating the bottom flange with an electric heater and cooling the top flange with fans. A symmetrical void position was achieved with the temperature difference between the flanges of $14-15 \mathrm{~K}$ for the $1.95 \mu \mathrm{m}$ diameter microparticles and $15-17 \mathrm{~K}$ for the $2.15 \mu \mathrm{m}$ diameter microparticles.

The microparticles were illuminated by a laser sheet with the wavelength of $532 \mathrm{~nm}$. Three Ximea MQ042RG-CM video cameras with interference bandpass filters captured the microparticle motion and the plasma emission in the same discharge area. The filters had the central wavelengths of 532, 750 , and $810 \mathrm{~nm}$, respectively, and the width of transmission band of the filters was about $10 \mathrm{~nm}$. Hence, one plasma glow observation camera captured the plasma radiation in 750.4 and $751.5 \mathrm{~nm}$ spectral lines, these lines correspond to the transitions $2 p_{1} \rightarrow 1 s_{2}$ and $2 p_{5} \rightarrow 1 s_{4}$ in the Paschen notation, respectively. Another camera captured the light in 810.4 and $811.5 \mathrm{~nm}$ spectral lines, which correspond to the transitions $2 p_{7} \rightarrow 1 s_{4}$ and $2 p_{9} \rightarrow 1 s_{5}$, respectively. The spatial resolution of the cameras was about $37 \mu \mathrm{m} /$ pixel. The frame rate of the cameras was $200 \mathrm{fps}$. The video capture of all three cameras was started by the software approximately at the same time. The difference between the time stamps of the frames with the same number was less than the duration of a single frame. All three cameras were focused on the central cross section of the discharge chamber.

We used the optogalvanic effect $[36,37]$ induced by the Toptica DL Pro laser for the investigation of the void stability [26]. The width of the laser spectral line was less than $1 \mathrm{MHz}$. The power of the laser beam entering the plasma chamber was about $50 \mathrm{~mW}$, and its diameter was $2 \mathrm{~mm}$. The laser was tuned to the $772.38 \mathrm{~nm}$ transition of argon and scanned the spectral range of about $220 \mathrm{MHz}$ in the vicinity of its center 


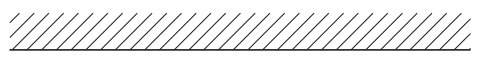
ionization $E=15.76 \mathrm{eV}$

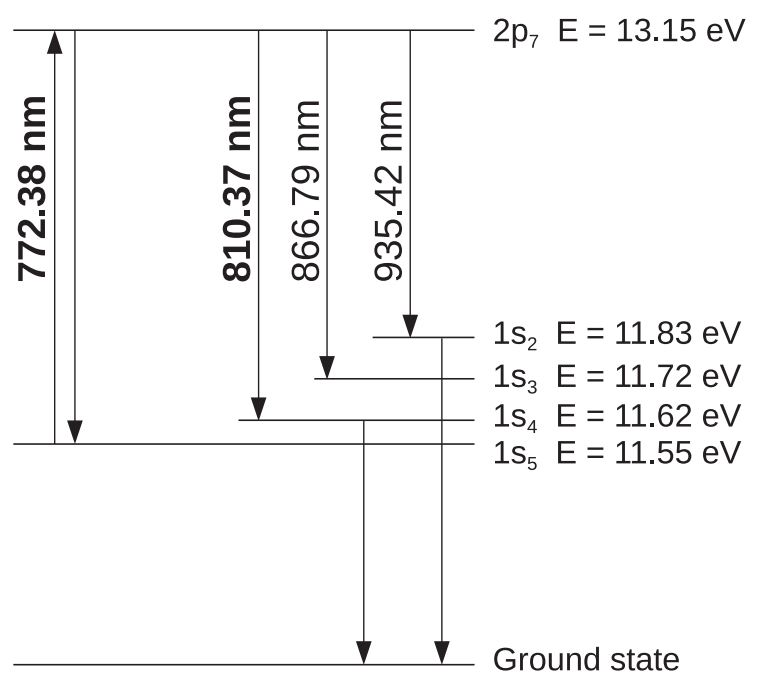

FIG. 2. Electronic levels of argon involved in the optogalvanic effect. The laser pumps the transition at $772.38 \mathrm{~nm}$ and fluorescence in the $810.37-\mathrm{nm}$ spectral line is observed.

with the repetition frequency of $100 \mathrm{~Hz}$. A small fraction of the laser light passed though a reference argon lamp to control the scanning range. A mechanical chopper was used to modulate the laser beam. The laser light induced the fluorescence in the $810.4 \mathrm{~nm}$ spectral line (see Fig. 2), which was used to match the laser modulation with the video frames.

At the beginning of every experiment, after stabilization of the temperature gradient, we set the discharge power to $500 \mathrm{~mW}$ and injected the microparticles till the onset of the heartbeat instability. The parameters of the instability, such as its frequency, the threshold discharge power of the instability onset, and necessary laser parameters for the optogalvanic control, drifted on the time scales of minutes making the measured quantities difficult to reproduce. Such evolution of the conditions is typical for complex plasma experiments [38]. However, the qualitative effects reported in this paper are well reproducible.

\section{RESULTS}

\section{A. Self-excited instability}

The self-excited heartbeat instability existed within a certain range of the discharge power and the gas pressure and required relatively high density of the microparticle suspension. An example of the heartbeat self-excitation region in pressure-discharge power space was published in Ref. [26]. This region was expanding with the increase of the amount of microparticles.

At the lower discharge power boundary of the instability range, the void underwent sporadic contractions with strong variation of the void size, while near the higher power boundary, the void size variation was decreasing with the increase of the discharge power, until the oscillations became undetectable. For example, in one experiment with the mi- croparticle diameter of $2.15 \mu \mathrm{m}$, the heartbeat appeared as the sporadic contractions at the discharge power of $500 \mathrm{~mW}$ with the variation of the void cross-section area between 31 and 7 $\mathrm{mm}^{2}$. After the discharge power was increased to $750 \mathrm{~mW}$, the void cross-section area oscillated between 30 and $27 \mathrm{~mm}^{2}$ with the frequency of $26 \mathrm{~Hz}$. In other our experiments, the lower discharge power of the heartbeat self-excitation was 300-600 mW, and the oscillations became invisible for the discharge powers higher than $800 \mathrm{~mW}$.

The reported experiments were conducted near the lower power boundary, where the instability onset had the threshold character and the void oscillations were well pronounced. In the experiments with the self-exited heartbeat instability, the amount of microparticles only slightly exceeded the instability onset threshold.

Similarly to Ref. [21], we obtained the spatiotemporal distributions (periodograms) of the microparticle velocity. First, we calculated the velocities $v_{x}$ using OpenPIV code [39] in cells $32 \times 32$ pixels with $50 \%$ overlap. Then, for every frame, we averaged the velocities along the vertical direction in a horizontal stripe with the width of four cells. The stripe was located at the height of the void [see Fig. 3(a)]. The obtained horizontal velocity profiles were stacked into a spatiotemporal distribution [see an example in Fig. 3(c)].

In a similar way, $x$ and $y$ distributions of emission intensity were obtained by stacking the profiles of the averaged emission intensities across the respective narrow stripes. The directions of $x$ and $y$ axes are shown in Fig. 1(a). In order to obtain the emission variation, the time-averaged intensity was subtracted. The emission variations for the filter with the central wavelength of $750 \mathrm{~nm}$ in horizontal and vertical directions $\left(I_{750}^{x}\right.$ and $I_{750}^{y}$, respectively) are presented in Fig. 3(c). Just before the void contraction, the plasma glow shortly flashed in the void [see Figs. 3(b) and 3(c) and movie_fig3.avi in Supplemental Material [40]]. The intensity at the flash maximum was $30-40 \%$ higher than the time-averaged value. At the same time, the discharge edge regions became darker. After that, the emission decreased in the contracted void, whereas the emission from the edge regions increased above the level before the flash. This effect was already reported in several works [23-26]. The plasma emission measured with 750- and 810 -nm filters behaved similarly. If the amount of microparticles in the suspension was slightly below the instability onset threshold, the spatial distribution of plasma emission was diffuse. Therefore, in this case, the void was in the dim regime (in the terminology of Ref. [33]).

Small oscillations of the microparticle motion and the plasma emission are visible in the spatiotemporal distributions between the large void collapses. They were termed "failed contractions" in Refs. [24,25]. In the velocity spatiotemporal distributions, a small slope of the oscillations can be seen, which suggests that the oscillation propagated from the discharge edges to the center. In our paper, we would therefore refer to them as to "breathing oscillations".

\section{B. Optogalvanic control}

We will describe the effects of the optogalvanic control of the heartbeat instability using the three spatiotemporal distributions: the distributions of $v_{x}$ and $I_{750}^{x}$ as in Fig. 3(c) and 

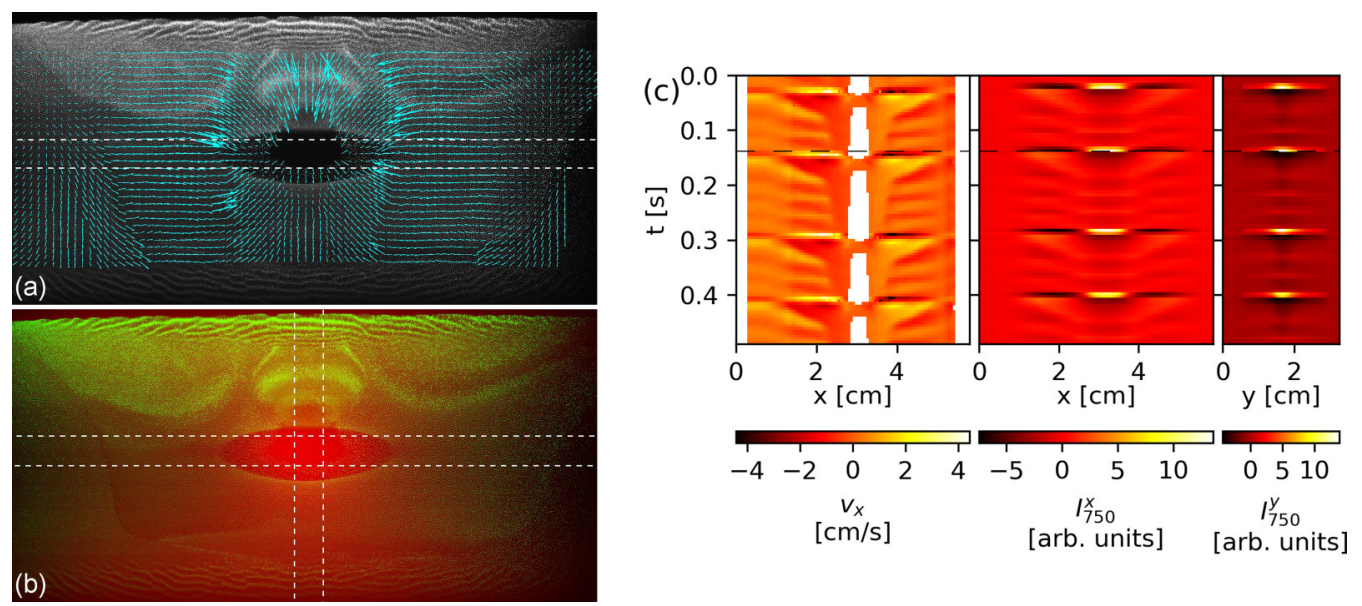

FIG. 3. Self-excited heartbeat instability. (a) One of the frames with the velocity field reconstructed using the OpenPIV software [39]. (b) The same frame with superimposed images of (green) the microparticle suspension and (red) the plasma emission captured with the filter with the central wavelength of $750 \mathrm{~nm}$. The dashed lines in plates (a) and (b) depict the areas used for the calculation of the spatiotemporal distributions. (c) Spatiotemporal distributions of the velocity $v_{x}$ and the plasma emission variations $I_{750}^{x}$ and $I_{750}^{y}$ during the self-excited heartbeat instability. The white color in the $v_{x}$ plot depicts the areas in which no microparticles are present. The black dashed lines depict the temporal position of the frames shown in plates (a) and (b). The microparticle diameter was $2.15 \mu \mathrm{m}$, the discharge power was $400 \mathrm{~mW}$. See also Supplemental Material [40].

additionally $x$ distribution of the emission intensity variation for the filter with the $810-\mathrm{nm}$ central wavelength $I_{810}^{x}$. The $I_{810}^{x}$ distribution shows the periods when the laser was open or closed in the experiments with the modulated laser due to the laser-induced fluorescence (see Fig. 2). In all cases, these distributions were obtained in the same way using the technique described in Sec. III A.

\section{Continuous laser}

The continuous laser beam passing through the void stabilized the microparticle suspension. Without the laser, the heartbeat instability was present in the suspension. The frequency of the heartbeat instability in that case was $17.9 \mathrm{~Hz}$ [see Fig. 4(a)]. We modulated the laser with the frequency of 3.6 Hz. While the laser was closed, the heartbeat instability was observed in the suspension. However, once the laser was opened, the instability disappeared and only breathing oscillations being slowly damped remained visible. This effect is demonstrated in Fig. 4(b) and movie_4b.avi in the Supplemental Material [40]. The continuous laser beam stabilized the void completely, and no oscillations were visible [see Fig. 4(c)].

\section{Transient stimulation}

The laser beam could transiently, either on its opening or closing, excite the heartbeat instability in a stable microparticle suspension. If the laser beam passed through the void, the void collapse occurred just after the beam closing. The spatiotemporal distributions of the transient heartbeat stimulation are presented in Fig. 5 and corresponding movies are available in Supplemental Material [40]. In the case of the chopper frequency of $1.7 \mathrm{~Hz}$ [Fig. 5(a)], every closing of the beam caused the void collapse. Also, small breathing oscillations quickly damped after opening the beam are visible. For the chopper frequencies of $19.6 \mathrm{~Hz}$ [Fig. 5(b)] and $44.0 \mathrm{~Hz}$ [Fig. 5(c)], the void collapses occurred every two and every four laser pulses, respectively. It seems the void needs to reach a certain phase of expansion after the collapse to be ready for a new stimulated contraction.

At the same time, the chopper frequency must not be too low. In one experiment, the transiently induced contractions occurred with the chopper frequency of $4.6 \mathrm{~Hz}$, but did not appear with the chopper frequency of $3 \mathrm{~Hz}$.

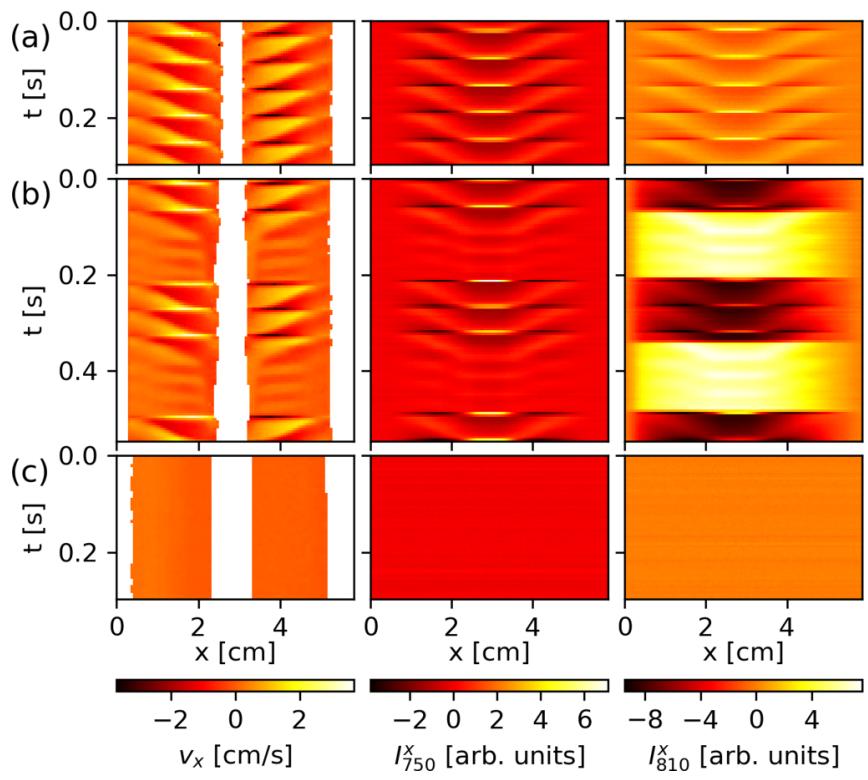

FIG. 4. The horizontal spatiotemporal distributions of the velocities $v_{x}$ and the plasma emission variations $I_{750}^{x}$ and $I_{810}^{x}$ (a) without the laser, (b) with the modulated laser, and (c) with continuous laser. The diameter of the microparticles was $1.95 \mu \mathrm{m}$, the discharge power was $500 \mathrm{~mW}$. See also Supplemental Material [40]. 


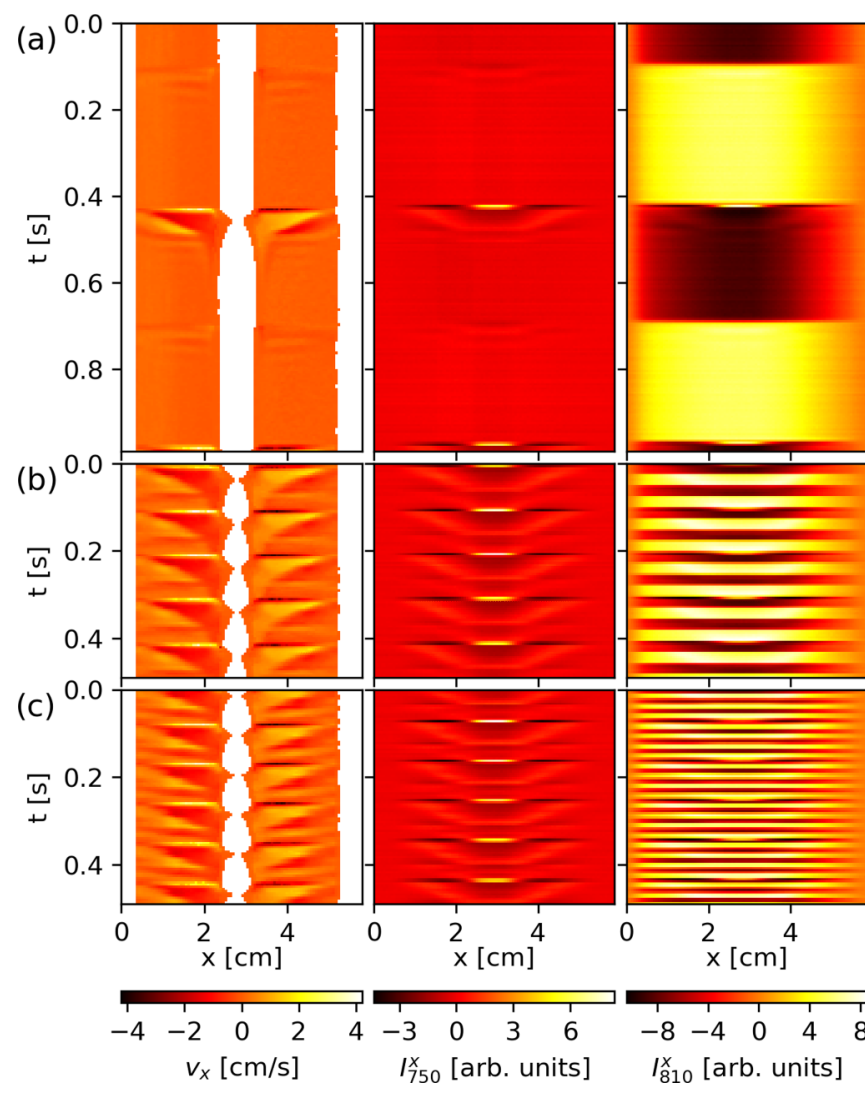

FIG. 5. Transient optogalvanic heartbeat excitation with the chopper frequency of (a) $1.7 \mathrm{~Hz}$, (b) $19.6 \mathrm{~Hz}$, (c) $44.0 \mathrm{~Hz}$. The microparticle diameter was $1.95 \mu \mathrm{m}$, the discharge power was $500 \mathrm{~mW}$. See also Supplemental Material [40].

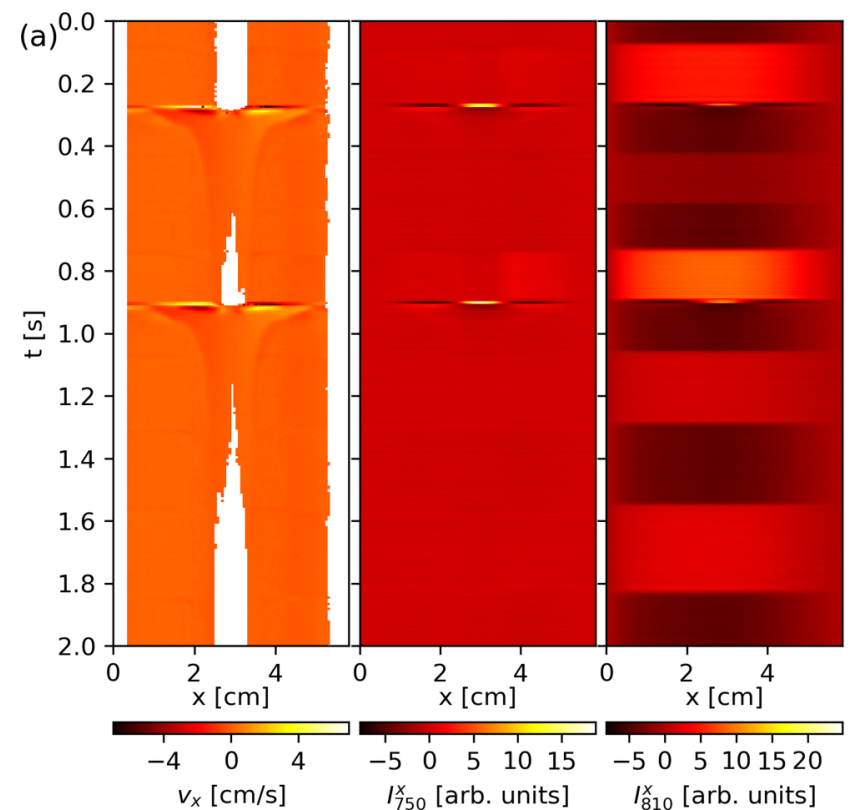

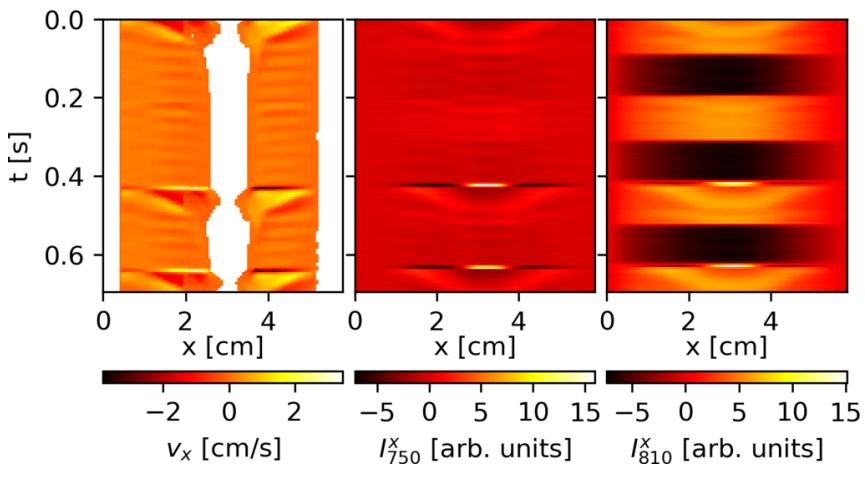

FIG. 7. Effect of the beam horizontal shift by $10 \mathrm{~mm}$. The microparticle diameter was $2.15 \mu \mathrm{m}$, the discharge power was $500 \mathrm{~mW}$.

To evaluate how the effect depends on the laser intensity, we equipped the chopper with neutral density filters of different transmission. The experiments showed that the further the suspension is from the self-excitation threshold, the higher laser power is necessary to stimulate the contraction. An example of the results is presented in Fig. 6. In this experiment, the measurements with the discharge power of $250 \mathrm{~mW}$ were performed $6 \mathrm{~min}$ after the measurements with the discharge power of $350 \mathrm{~mW}$ without injection of the microparticles in between.

If the laser beam was shifted horizontally from the void center to the distance of $1 \mathrm{~cm}$, the void collapsed after the laser opening (see Fig. 7) in contrast with the experiments where the unshifted laser beam caused the void contraction after closing. We could not obtain this change of the void collapse phase with a vertical shift of the laser beam: the effect of the laser beam under or above the void became weaker, as it was reported in Ref. [26], and only rare void

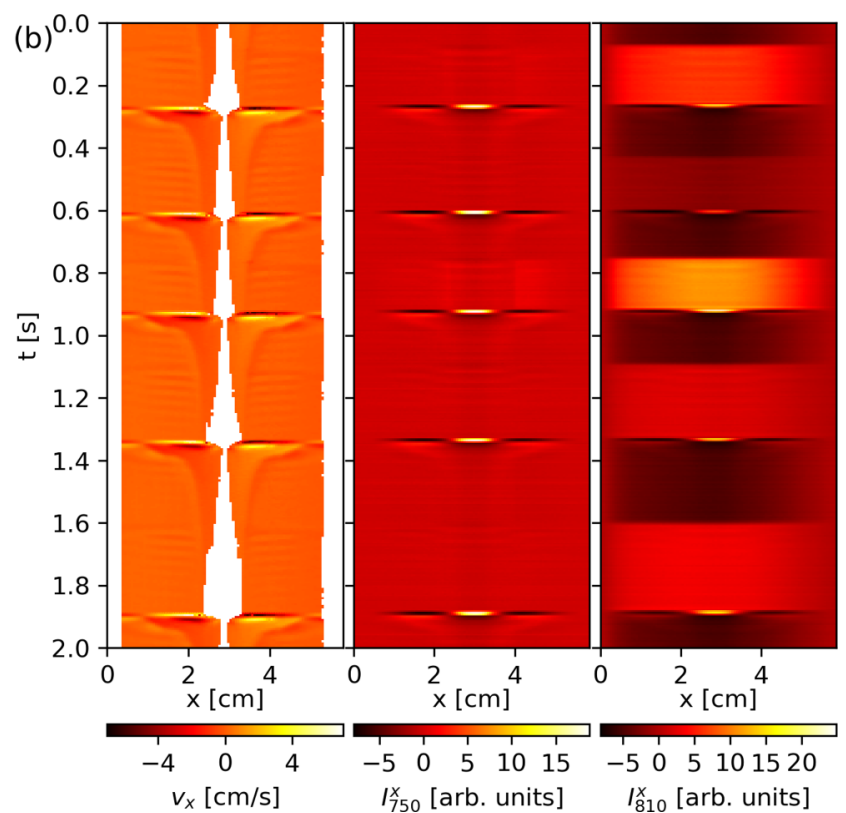

FIG. 6. Transient optogalvanic heartbeat excitation with the attenuated laser beam. The microparticle diameter was $1.95 \mu \mathrm{m}$, the discharge power was (a) $250 \mathrm{~mW}$, (b) $350 \mathrm{~mW}$. The bright stripes in the $I_{810}^{x}$ distribution correspond to the time intervals when the laser was crossing the void through the filters with the following values of the transmission (from the top to the bottom): $0.45,0.014,1,0.12$, and 0.19 . 
contractions occurred, still after the laser closing. This difference between the horizontal and vertical shifts of the beam corresponds to the difference between horizontal and vertical spatiotemporal emission distributions during the flash [see Fig 3(c)].

\section{Resonant stimulation}

If the void contractions could not be induced transiently, the heartbeat instability could be stimulated resonantly. This effect was reported in Ref. [26]. It was discovered using the same laser and discharge chamber as in this work. An example of this effect is shown in Fig. 8. In the case of the lowest chopper frequency [Fig. 8(a)], small breathing oscillations, whose phase is disturbed by the modulated laser beam, are visible. Horizontally averaged Fourier spectra of the spatiotemporal distributions for this case are shown in Fig. 9(a). In this spectrum, mainly two modes are visible: The first one with the frequency of about $10 \mathrm{~Hz}$ corresponds to the laser modulation, whereas the second one with the frequency of about $29 \mathrm{~Hz}$ corresponds to the breathing oscillations. At higher chopper frequency, the microparticle suspension oscillated with the frequency of the laser modulation and low amplitude [Fig. 8(b)]. At the chopper frequencies close to the frequency of the breathing oscillations, the heartbeat instability occurred [see Figs. 8(c)-8(e) and movie_fig8d.avi in the Supplemental Material [40]]. The width of this resonance was several Hz.

The Fourier spectra for the spatiotemporal distributions in Fig. 8(d) are shown in Fig. 9(b). Mixing of the modulation frequency of $27.3 \mathrm{~Hz}$ with four times lower heartbeat frequency of $6.8 \mathrm{~Hz}$ leads to the frequency comb spectrum [41]. We note that the spectrum of the heartbeat instability observed in Refs. [13,23] also has a frequency comb character.

\section{DISCUSSION}

\section{A. Dim-to-bright void transition and void collapse}

In Refs. [26], the heartbeat instability was supposed to occur due to a critical transformation on the void boundary from a smooth potential profile to the sheath. The sheath was suggested to appear when the microparticle number density reaches a certain value: When the amount of microparticles is small, they do not significantly disturb the plasma, and the potential profile across the void boundary should be smooth. If the suspension is however dense, the void boundary should act similarly to a solid surface, in front of which the sheath potential drop should occur. However, simulation with a 1D hybrid model and fixed microparticle distribution showed a linear growth of the electric field with increasing the microparticle number density without any critical phenomena [42]. According to recent findings [33], a steep growth of the electric field accompanies the transition between dim and bright void regimes. Since the heartbeat instability is accompanied by the flash inside the void, it is reasonable to suppose that the void contractions are caused by an abrupt transition between dim and bright void regimes.

This process is fast in comparison with the microparticle motion: In Ref. [26] the flash was measured with a photomul-
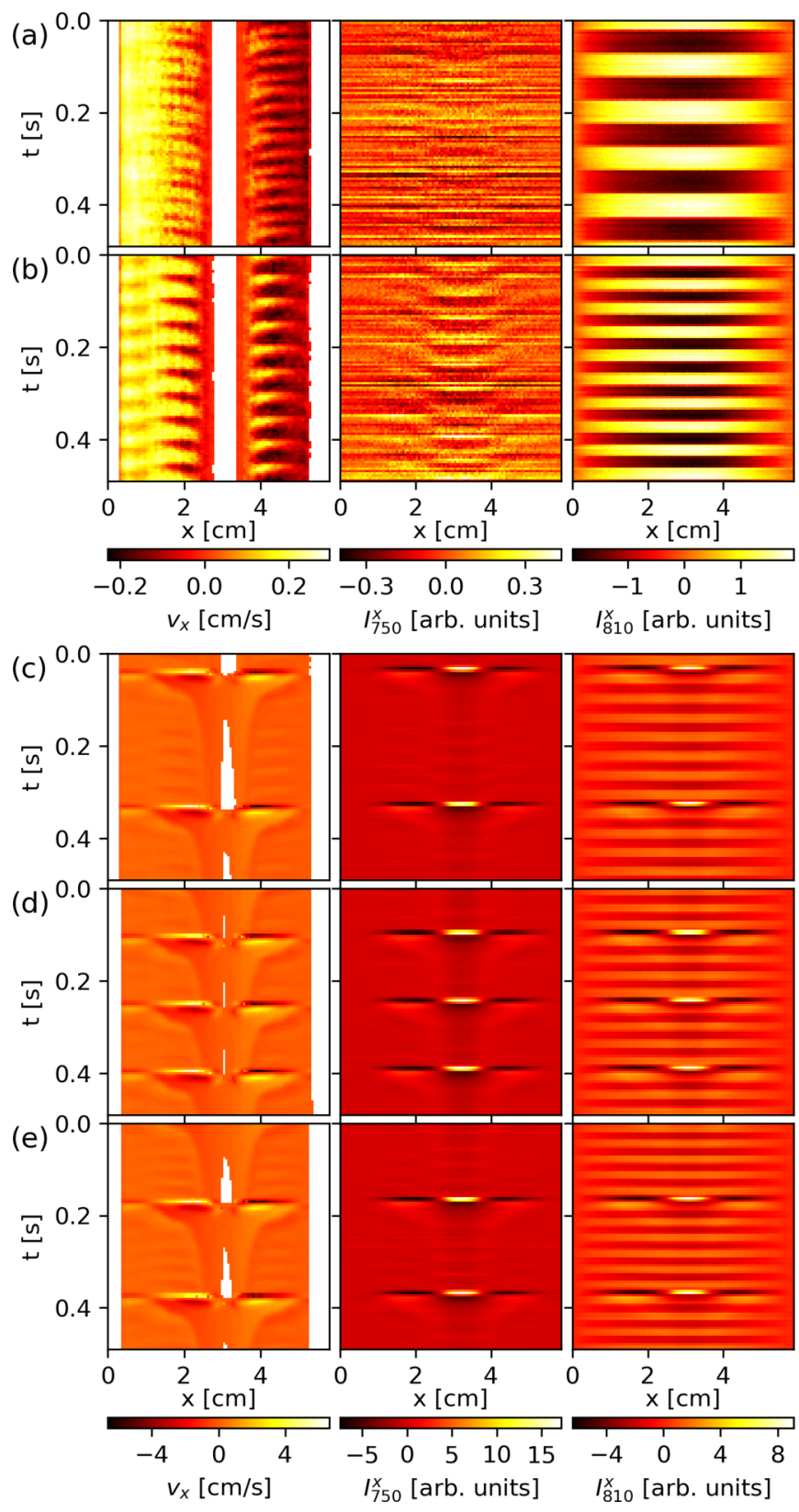

FIG. 8. Stimulation of the heartbeat instability with low laser power. The laser beam was attenuated down to $0.7 \mathrm{~mW}$. The microparticle diameter was $2.15 \mu \mathrm{m}$, the discharge power was 350 $\mathrm{mW}$. The laser modulation frequency was (a) $9.8 \mathrm{~Hz}$, (b) $19.5 \mathrm{~Hz}$, (c) $23.9 \mathrm{~Hz}$, (d) $27.3 \mathrm{~Hz}$, and (e) $29.5 \mathrm{~Hz}$. See also Supplemental Material [40].

tiplier and looked as a pulse with the duration of $5 \mathrm{~ms}$ and steep edges. In the experiments with growing nanoparticles, the flash duration of $2 \mathrm{~ms}$ was reported [24]. It leads therefore to the transient formation of a bright-void-like emission (and, consequently, ionization) profile under the plasma conditions of a dim void. Similar situation was considered in Ref. [32] using a 1D hybrid model: Simulations with a fixed microparticle distribution with a void resulted in a strong emission inside the void and uncompensated electrostatic forces pushing the microparticles to the void center. 

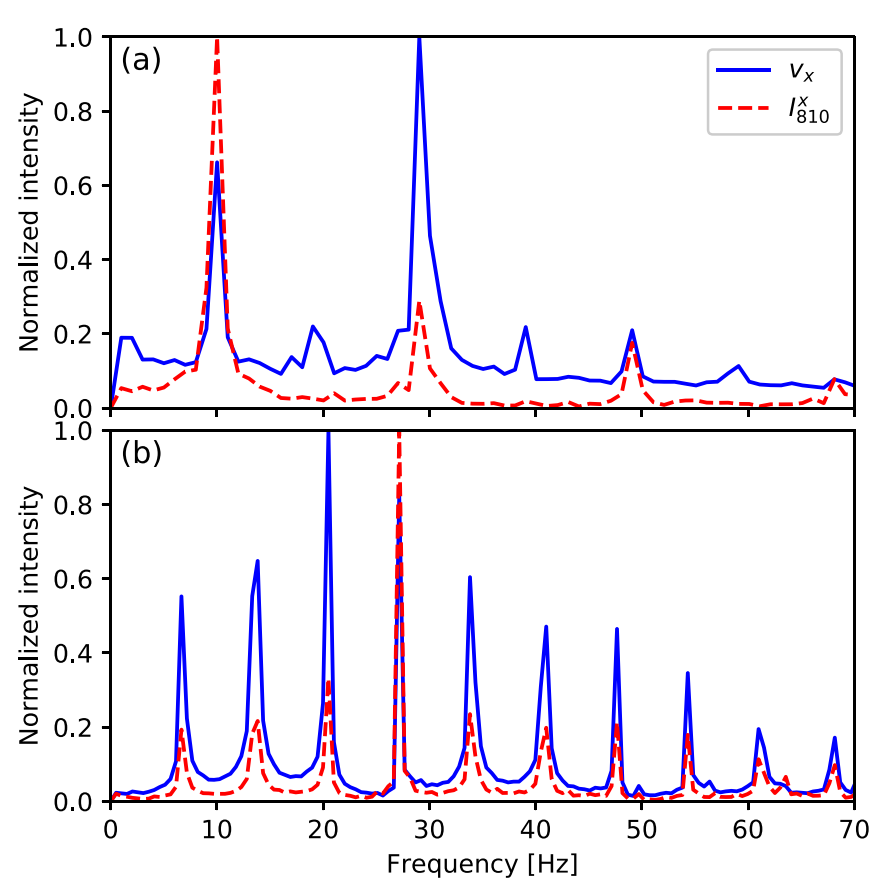

FIG. 9. Horizontally averaged Fourier spectra of $v_{x}$ and $I_{810}^{x}$ spatiotemporal distributions shown in (a) Fig. 8(a); (b) Fig. 8(d). The laser modulation frequency was (a) $9.8 \mathrm{~Hz}$, (b) $27.3 \mathrm{~Hz}$. Every spectrum is normalized to its maximal value.

\section{B. Optogalvanic effect}

In Ref. [33], it was pointed out that an increase of the ionization rate may switch the void from the dim to bright regime. The laser whose wavelength is tuned to the electron transition of the plasma forming gas atoms is capable of modifying the ionization rate in the plasma. This is the underlying mechanism of the optogalvanic effect $[43,44]$. The ionization energy of argon from the ground state is $15.76 \mathrm{eV}$ [45], which is much higher than the electron temperature in our plasma $\left(T_{e} \sim 4 \mathrm{eV}, n_{e} \sim 10^{15} \mathrm{~m}^{-3}\right.$ [32,33]). The bottom state of the transition pumped by the laser is the lowest metastable level, which lies only $4.21 \mathrm{eV}$ below the ionization energy. Although, after additional excitation by the laser, the atom can be ionized even easier, it can spontaneously relax to the ground state (see Fig. 2). The optogalvanic effect is therefore determined by the interplay between the growth of the ionization rate due to the increase in the population of the upper level of the laser-pumped transition and the decrease of the ionization due to the decrease in the population of the lower level of the laser-pumped transition. The sign of the effect depends on the experimental conditions. For example, nanosecond laser pulses in the $696.5 \mathrm{~nm}$ argon spectral line caused a decrease of ionization in a glow discharge [43], whereas much longer pulses $(0.4 \mathrm{~ms})$ in the same spectral line increased the ionization in a RF discharge [44].

In our case, the probability of the relaxation to $1 s_{2}$ and $1 s_{4}$ resonant states is one order of magnitude higher than the probability to relax to the $1 s_{3}$ metastable state [46]. According to Ref. [43], a laser pulse in $772.38 \mathrm{~nm}$ argon spectral line caused a positive pulse of DC discharge voltage (at constant discharge current), which implies the decrease of the ionization rate. The laser-induced decrease of the ionization rate is consistent with the stabilization of the heartbeat instability by the continuous laser since the dim void condition corresponds to the lower ionization rate [33].

\section{Laser control of the heartbeat instability}

In the case of self-excited heartbeat instability, the transition from the dim to bright void regime is evidently mediated by the breathing oscillations: They are always seen as precursors of the heartbeat instability as pointed out also in Refs. [13,24]. The dim void formation requires significant radial ion losses [33], which could be modulated by the breathing oscillations. In the case of resonant optogalvanic stimulation of the heartbeat instability, the laser slightly modulates the ionization rate (as pointed out in Ref. [26]). The heartbeat instability is excited when the modulation frequency is close to the frequency of the breathing oscillations. Also, the breathing oscillations are damped, once the heartbeat instability is switched off by the continuous laser beam [see Fig. 4(c)].

During the transient heartbeat stimulation, the transition from dim to bright void occurs immediately after opening or closing the laser beam independent of the existence or phase of the breathing oscillations. We suppose that in this case, the ionization rate inside the void increases due to the transient processes in the plasma. If the laser beam passes though the void, its closing causes this increase directly. If the beam is shifted, a decrease of the ionization near the beam requires an ionization increase in other parts of the discharge.

\section{CONCLUSION}

The presented experiments reproduced earlier reported behavior of the plasma emission during the heartbeat instability, small breathing oscillations that appear in the suspension between the void contractions, and resonant optogalvanic stimulation of the instability. It was demonstrated that the resonance is observed when the laser modulation frequency coincides with the frequency of these breathing oscillations. The experiments with high laser power revealed that individual laser switching can cause the void contraction transiently. The void contracts after closing of the laser beam if it passes through the void center, and after opening of the laser beam if it was shifted horizontally to the void periphery. However, continuous laser beam stabilizes the microparticle suspension.

The experimental results allow us to describe the cycle of the heartbeat instability in the following way (Fig. 10): It starts from a microparticle suspension with a dim void. The void transits abruptly to the bright regime due to the breathing oscillations, whose amplitude grows beyond a critical value [24]. The transition can also be induced by a steep change of ionization rate by the laser or the laser working in resonance with the breathing oscillations. In the bright regime, the void boundary becomes mechanically unstable and the void collapses due to large electrostatic forces on its boundaries. After the collapse, the microparticles absorb the surplus of electrons and ions and move back restoring the dim-void configuration.

We were, therefore, able to identify a sequence of physical conditions the plasma acquires during the cycle of the heartbeat instability (green boxes in Fig. 10). However, most of 


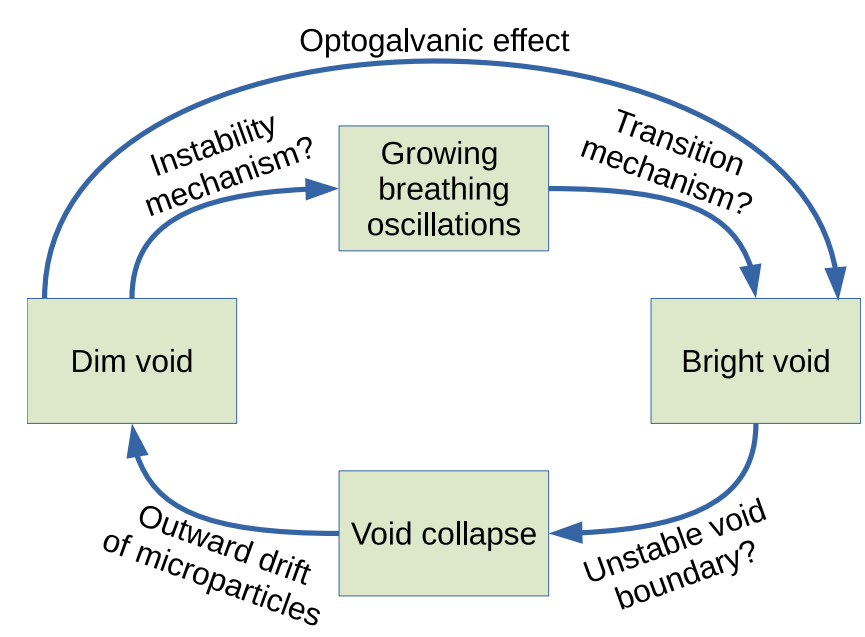

FIG. 10. Schematic representation of the heartbeat instability cycle. Initially dim void transits to the bright regime. This transition is mediated either by the breathing-oscillations-related mechanism or by the transient optogalvanic effect. The boundary of the bright void becomes unstable and the void collapses due to the electrostatic force prevailing over the ion drag and thermophoretic forces. The dim void recovers due to the outward drift of microparticles.

the transitions between them (arrows in Fig. 10) are still not clear. What is the self-excitation mechanism of the breathing oscillations in the dim void regime? How does their growth lead to the abrupt transition to the bright void regime? Why does the void boundary in the bright regime get mechanically unstable? What makes the microparticles move backward and form the dim void after the bright void collapse? In the discussion we suggested hypothetical mechanisms which could explain some of these transitions. They need to be proved by further investigations, theoretical as well as experimental.

As we already mentioned, the heartbeat instability is a multi-time-scale process. Its direct simulation is, therefore, computationally very expensive. Decomposing the heartbeat into stages allows us to model each of them individually. The optogalvanic control allows us to experimentally separate the void collapse from the breathing oscillations. This approach could be applied to other instabilities in complex plasmas.

\section{ACKNOWLEDGMENTS}

We thank Dr. V. Nosenko for careful reading of our manuscript. The PK-3 Plus chamber was funded by the space agency of the German Aerospace Center (DLR) with funds from the federal ministry for economy and technology according to a resolution of the Deutscher Bundestag under Grants No. 50WP0203 and No. 50WM1203. A. P. acknowledges the financial support of Deutscher Akademischer Austauschdienst (DAAD) with funds from DLR.
[1] V. E. Fortov, A. V. Ivlev, S. A. Khrapak, A. G. Khrapak, and G. E. Morfill, Complex (dusty) plasmas: Current status, open issues, perspectives, Phys. Rep. 421, 1 (2005).

[2] G. E. Morfill and A. V. Ivlev, Complex plasmas: An interdisciplinary research field, Rev. Mod. Phys. 81, 1353 (2009).

[3] A. Ivlev, H. Löwen, G. Morfill, and C. P. Royall, Complex Plasmas and Colloidal Dispersions: Particle-Resolved Studies of Classical Liquids and Solids, Series in Soft Condensed Matter (World Scientific, Singapore, 2012), Vol. 5.

[4] I. Pilch and F. Greiner, Diagnostics of void expansion during cyclic growth and formation of layered nanoparticle clouds, J. Appl. Phys. 121, 113302 (2017).

[5] R. L. Merlino, 25 years of dust acoustic waves, J. Plasma Phys. 80, 773 (2014).

[6] A. V. Zobnin, A. D. Usachev, A. M. Lipaev, O. F. Petrov, V. E. Fortov, M. Y. Pustylnik, H. M. Thomas, M. A. Fink, M. H. Thoma, and G. I. Padalka, Transverse ionization instability of the elongated dust cloud in the gas discharge uniform positive column under microgravity conditions, J. Phys.: Conf. Ser. 774, 012174 (2016), XXXI International Conference on Equations of State for Matter (ELBRUS 2016) 1-6 March 2016, Elbrus, Russia.

[7] G. Praburam and J. Goree, Experimental observation of very low-frequency macroscopic modes in a dusty plasma, Phys. Plasmas 3, 1212 (1996).

[8] D. Samsonov and J. Goree, Instabilities in a dusty plasma with ion drag and ionization, Phys. Rev. E 59, 1047 (1999).

[9] M. Mikikian, M. Cavarroc, L. Couëdel, and L. Boufendi, Low frequency instabilities during dust particle growth in a radiofrequency plasma, Phys. Plasmas 13, 092103 (2006).
[10] M. Mikikian, H. Tawidian, and T. Lecas, Merging and Splitting of Plasma Spheroids in a Dusty Plasma, Phys. Rev. Lett. 109, 245007 (2012).

[11] M. Mikikian, L. Couedel, Y. Tessier, and L. Boufendi, Carousel instability in a capacitively coupled rf dusty plasma, IEEE Trans. Plasma Sci. 39, 2748 (2011).

[12] J. Goree, R. Quinn, G. Morfill, H. Thomas, T. Hagl, U. Konopka, H. Rothermel, and M. Zuzic, Plasma dust crystallization, in Proceedings of the 4th Microgravity Fluid Physics and Transport Phenomena Conference, Cleveland (NASA, 1998), pp. 105-106.

[13] M. Mikikian, M. Cavarroc, L. Couëdel, Y. Tessier, and L. Boufendi, Mixed-Mode Oscillations in Complex-Plasma Instabilities, Phys. Rev. Lett. 100, 225005 (2008).

[14] M. Brøns, T. J. Kaper, and H. G. Rotstein, Introduction to focus issue: Mixed mode oscillations: Experiment, computation, and analysis, Chaos 18, 015101 (2008).

[15] T. Braun, J. A. Lisboa, and J. A. C. Gallas, Evidence of Homoclinic Chaos in the Plasma of a Glow Discharge, Phys. Rev. Lett. 68, 2770 (1992).

[16] T. Hayashi, Mixed-Mode Oscillations and Chaos in a Glow Discharge, Phys. Rev. Lett. 84, 3334 (2000).

[17] F. N. Albahadily, J. Ringland, and M. Schell, Mixed-mode oscillations in an electrochemical system. I. A Farey sequence which does not occur on a torus, J. Chem. Phys. 90, 813 (1989).

[18] V. Petrov, S. K. Scott, and K. Showalter, Mixed-mode oscillations in chemical systems, J. Chem. Phys. 97, 6191 (1992).

[19] J. Rubin and M. Wechselberger, Giant squid-hidden canard: The 3D geometry of the Hodgkin-Huxley model, Biol. Cybern. 97, 5 (2007). 
[20] B. van der Pol D.Sc. and J. van der Mark, LXXII. The heartbeat considered as a relaxation oscillation, and an electrical model of the heart, Philos. Mag. J. Sci. 6, 763 (1928).

[21] S. K. Zhdanov, M. Schwabe, R. Heidemann, R. Sütterlin, H. M. Thomas, M. Rubin-Zuzic, H. Rothermel, T. Hagl, A. V. Ivlev, G. E. Morfill, V. I. Molotkov, A. M. Lipaev, O. F. Petrov, V. E. Fortov, and T. Reiter, Auto-oscillations in complex plasmas, New J. Phys. 12, 043006 (2010).

[22] R. J. Heidemann, L. Couëdel, S. K. Zhdanov, K. R. Sütterlin, M. Schwabe, H. M. Thomas, A. V. Ivlev, T. Hagl, G. E. Morfill, V. E. Fortov, V. I. Molotkov, O. F. Petrov, A. I. Lipaev, V. Tokarev, T. Reiter, and P. Vinogradov, Comprehensive experimental study of heartbeat oscillations observed under microgravity conditions in the PK-3 Plus laboratory on board the International Space Station, Phys. Plasmas 18, 053701 (2011).

[23] M. Mikikian and L. Boufendi, Experimental investigations of void dynamics in a dusty discharge, Phys. Plasmas 11, 3733 (2004).

[24] M. Mikikian, L. Couëdel, M. Cavarroc, Y. Tessier, and L. Boufendi, Self-excited void instability in dusty plasmas: Plasma and dust cloud dynamics during the heartbeat instability, New J. Phys. 9, 268 (2007).

[25] M. Mikikian, L. Couëdel, M. Cavarroc, Y. Tessier, and L. Boufendi, Threshold Phenomena in a Throbbing Complex Plasma, Phys. Rev. Lett. 105, 075002 (2010).

[26] M. Y. Pustylnik, A. V. Ivlev, N. Sadeghi, R. Heidemann, S. Mitic, H. M. Thomas, and G. E. Morfill, On the heterogeneous character of the heartbeat instability in complex (dusty) plasmas, Phys. Plasmas 19, 103701 (2012).

[27] M. Kretschmer, S. A. Khrapak, S. K. Zhdanov, H. M. Thomas, G. E. Morfill, V. E. Fortov, A. M. Lipaev, V. I. Molotkov, A. I. Ivanov, and M. V. Turin, Force field inside the void in complex plasmas under microgravity conditions, Phys. Rev. E 71, 056401 (2005).

[28] L. Couëdel, M. Mikikian, A. A. Samarian, and L. Boufendi, Self-excited void instability during dust particle growth in a dusty plasma, Phys. Plasmas 17, 083705 (2010).

[29] S. V. Vladimirov, V. N. Tsytovich, and G. E. Morfill, Stability of dust voids, Phys. Plasmas 12, 052117 (2005).

[30] J. Goree, G. E. Morfill, V. N. Tsytovich, and S. V. Vladimirov, Theory of dust voids in plasmas, Phys. Rev. E 59, 7055 (1999).

[31] A. M. Lipaev, S. A. Khrapak, V. I. Molotkov, G. E. Morfill, V. E. Fortov, A. V. Ivlev, H. M. Thomas, A. G. Khrapak, V. N. Naumkin, A. I. Ivanov, S. E. Tretschev, and G. I. Padalka, Void Closure in Complex Plasmas under Microgravity Conditions, Phys. Rev. Lett. 98, 265006 (2007).

[32] M. Y. Pustylnik, I. L. Semenov, E. Zähringer, and H. M. Thomas, Capacitively coupled rf discharge with a large amount of microparticles: Spatiotemporal emission pattern and microparticle arrangement, Phys. Rev. E 96, 033203 (2017).

[33] A. Pikalev, I. Semenov, M. Pustylnik, C. Räth, and H. Thomas, Dim and bright void regimes in capacitively coupled RF complex plasmas, Plasma Sources Sci. Technol. 30, 035014 (2021).

[34] H. M. Thomas, G. E. Morfill, V. E. Fortov, A. V. Ivlev, V. I. Molotkov, A. M. Lipaev, T. Hagl, H. Rothermel, S. A. Khrapak, R. K. Suetterlin, M. Rubin-Zuzic, O. F. Petrov, V. I. Tokarev, and S. K. Krikalev, Complex plasma laboratory PK-3 plus on the International Space Station, New J. Phys. 10, 033036 (2008).

[35] H. Rothermel, T. Hagl, G. E. Morfill, M. H. Thoma, and H. M. Thomas, Gravity Compensation in Complex Plasmas by Application of a Temperature Gradient, Phys. Rev. Lett. 89, 175001 (2002).

[36] J. E. M. Goldsmith and J. E. Lawler, Optogalvanic spectroscopy, Contemp. Phys. 22, 235 (1981).

[37] B. Barbieri, N. Beverini, and A. Sasso, Optogalvanic spectroscopy, Rev. Mod. Phys. 62, 603 (1990).

[38] C. Killer, F. Greiner, S. Groth, B. Tadsen, and A. Melzer, Longterm spatiotemporal evolution of the dust distribution in dusty argon rf plasmas, Plasma Sources Sci. Technol. 25, 055004 (2016).

[39] Z. J. Taylor, R. Gurka, G. A. Kopp, and A. Liberzon, Longduration time-resolved PIV to study unsteady aerodynamics, IEEE Trans. Instrum. Meas. 59, 3262 (2010); OpenPIV C++ with Qt frontend., Source code: https://github.com/OpenPIV/ openpiv-c--qt.

[40] See Supplemental Material at http://link.aps.org/supplemental/ 10.1103/PhysRevE.104.045212 for videos with the data that were used to obtain Figs. 3, 4(b), 5, and 8(d).

[41] R. Holzwarth, T. Udem, T. W. Hänsch, J. C. Knight, W. J. Wadsworth, and P. S. J. Russell, Optical Frequency Synthesizer for Precision Spectroscopy, Phys. Rev. Lett. 85, 2264 (2000).

[42] M. Y. Pustylnik, I. L. Semenov, and H. M. Thomas, Emission and microparticle arrangement in capacitavely coupled rf discharge, in Asia-Pacific Conference on Plasma and Terahertz. Science (Xi' an, China, 2018).

[43] J. R. Nestor, Optogalvanic spectra of neon and argon in glow discharge lamps, Appl. Opt. 21, 4154 (1982).

[44] D. E. Murnick, R. B. Robinson, D. Stoneback, M. J. Colgan, and F. A. Moscatelli, Optogalvanic signals from argon metastables in a rf glow discharge, Appl. Phys. Lett. 54, 792 (1989).

[45] L. Minnhagen, Spectrum and the energy levels of neutral argon, Ar I, J. Opt. Soc. Am. 63, 1185 (1973).

[46] W. L. Wiese, J. W. Brault, K. Danzmann, V. Helbig, and M. Kock, Unified set of atomic transition probabilities for neutral argon, Phys. Rev. A 39, 2461 (1989). 